\title{
Atomic structure of nanomaterials by resonant high-energy XRD
}

\author{
V. Petkov \\ Dept. of Physics, Central Michigan University, MI-48848, USA \\ petko1vg@cmich.edu
}

With current technology moving rapidly toward smaller scales, diverse nanomaterials are being produced in increasing numbers and explored for various useful applications. The atomic structure of these materials can be well characterized by high-energy x-ray diffraction (XRD) coupled to atomic pair distribution function (PDF) analysis. However, a single diffraction experiment on a nanomaterial composed of $n$ atomic species yields an atomic PDF, usually referred to as a total PDF, composed of $n(n+1) / 2$ chemically distinct partial PDFs, which renders its interpretation ambiguous. Using resonant high-energy XRD allows particular partial PDFs to be highlighted and others dimmed thus giving nanostructure studies very much needed chemical specificity. In the talk we will briefly introduce the technique, including instrumentation, data collection, reduction and interpretation, and give examples from studies on ferroelectric nanosized powders, nanoalloys, metal-oxide nanocomposites, and others.

Scheme 1. Atomic structure at functional interfaces inside metallic nanoparticles by resonant high-energy XRD. The example features the core@shell interface inside Ru@Pt nanoparticles used as catalysts for electrooxidation of methanol in fuel cells. HAADF image and refined 3D model of the nanoparticles are shown in the sketch below on the left and right, respectively.

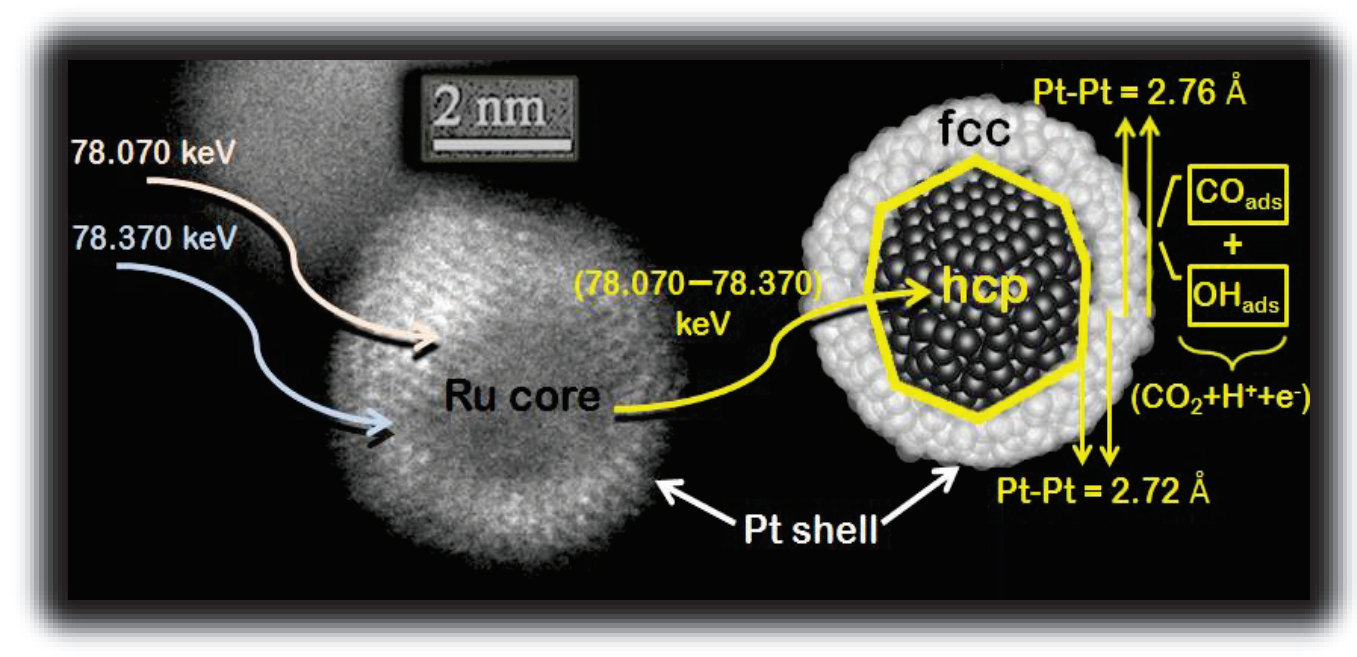

Two diffraction patterns are collected using X-rays with energy of $78.070 \mathrm{keV}$ and $78.370 \mathrm{keV}$ which are, respectively, 325 and $25 \mathrm{eV}$ below the $\mathrm{K}$ adsorption edge $(78.395 \mathrm{keV})$ of Pt atoms. The pattern obtained using X-rays with higher energy is subtracted from the one obtained using X-rays with lower energy. The intensity difference is due to the difference between the dispersion corrections to the atomic X-ray scattering factor of Pt atoms. Hence, the Fourier transform of the intensity, the so-called Pt-differential $\mathrm{PDF}$, reflects atomic pair correlations specific to $\mathrm{Pt}$ atoms, including $\mathrm{Pt}-\mathrm{Pt}$ bonding distances at the core@shell interface (see the vertical arrows). Experimental total and element specific PDFs allow testing and refining of 3D models for the nanoparticles, and then explore the relationship between their atomic structure and catalytic functionality. ${ }^{1}$

1. V. Petkov et al. ACS Appl. Mat. \& Interfaces 2015, 7, 23265-23277. 